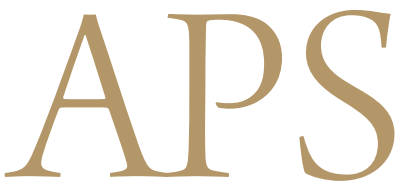

Archives of Plastic Surgery

\title{
Effect of Recombinant Human Epidermal Growth Factor Impregnated Chitosan Film on Hemostasis and Healing of Blood Vessels
}

\author{
Sangshin Lee ${ }^{1}$, Inwook Jung ${ }^{1}$, Seongcheol $\mathrm{Yu}^{2}$, Joon Pio Hong ${ }^{1}$ \\ ${ }^{1}$ Department of Plastic and Reconstructive Surgery, Asan Medical Center, University of Ulsan College of Medicine, Seoul; ${ }^{2}$ Department of \\ Plastic Surgery, Gangneung Asan Hospital, University of Ulsan College of Medicine, Gangneung, Korea
}

Background Bleeding can be a problem in wound debridement. In search for an effective hemostatic agent, we experimented with a chitosan film combined with the recombinant human epidermal growth factor (rh-EGF), hypothesizing that it would achieve effective hemostasis and simultaneously enhance arterial healing.

Methods Forty-eight Sprague-Dawley rats were used, and 96 puncture wounds were made. The wounds were divided into the following four groups: treated with sterile gauze, treated with gelatin sponge, treated with chitosan, and treated with chitosan combined with rh-EGF. Immediate hemostasis was evaluated, and arterial healing was observed histologically.

Results Groups B, C, and D showed a significant rate of immediate hemostasis as compared to group $A(P<0.05)$, but there were no significant differences among groups $B, C$ and $D$. Histologically, only group D showed good continuity of the vessel wall after 1 week. It was the only group to show smooth muscle cell nuclei of the vessel wall.

Conclusions We observed that chitosan has an effective hemostatic potential and the mix of rh-EGF and chitosan does not interfere with chitosan's hemostatic capabilities. We also identified enhanced healing of vessel walls when rh-EGF was added to chitosan. Further research based on these positive findings is needed to evaluate the potential use of this combination on difficult wounds like chronic diabetic ulcerations.

Keywords Epidermal growth factor / Chitosan / Hemostasis
Correspondence: Joon Pio Hong Department of Plastic and Reconstructive Surgery, Asan Medical Center, University of Ulsan College of Medicine, 88 Olympic-ro 43-gil,

Songpa-gu, Seoul 138-736, Korea

Tel: +82-2-3010-3600

Fax: +82-2-476-7471

E-mail: joonphong@amc.seoul.kr

Received: 1 Jan 2014 • Revised: 11 Apr 2014 • Accepted: 8 May 2014

pISSN: 2234-6163 • elSSN: 2234-6171 • http://dx.doi.org/10.5999/aps.2014.41.5.466• Arch Plast Surg 2014;41:466-471

No potential conflict of interest relevant to this article was reported.

\section{INTRODUCTION}

The treatment for any ulcer begins with debridement. Timely debridement, which removes all remaining foreign bodies and non-viable tissues, provides a healthy environment to start the wound-healing process. In cases with chronic wounds, the debridement process turns the wounds acute, allowing a re-ignition of the healing cascade. Bleeding occurs when performing an effective debridement. However, such bleeding can often be troubling as it can be prolonged, particularly in a patient with a diabetic foot ulcer, which may have an increased risk of bleeding, or in patients with chronic ulcers and on anti-platelet and anti-coagulant agents $[1,2]$. In most of the cases, hemostasis is achieved by compression, but there are still certain challenging cases with respect to bleeding control after debridement. These patients with bleeding tendencies may even influence the course 
of treatment when under the supervision of an inexperienced care provider.

Thus, the development of a new dressing material providing the properties for facilitating epithelization in addition to hemostasis against active bleeding may add value to the current passive dressing materials.

Chitosan has a positive electrical charge and draws the negatively charged red blood cells towards itself; it is also an effective inducer of platelet adhesion and aggregation [3,4]. It is known that increased actin polymerization and $\left[\mathrm{Ca}^{2+}\right] \mathrm{i}$ mobilization are involved in the regulation of platelet adhesion. Further, a chitosan dose independently increases the $\left[\mathrm{Ca}^{2+}\right] \mathrm{i}$ of platelets, suggesting that increased $\mathrm{Ca}^{2+}$ mobilization may be one of the important mechanisms leading to the activation of platelet function by chitosan [5]. This property may provide an insight into the development of chitosan into a new hemostatic material. Chitin, which is a raw material of chitosan, can be easily obtained from a crustacean's dermoskeleton, and its properties of biocompatibility, bioresolvability, and hemostatic effect have been reported [6,7]. Recombinant human epidermal growth factor (rh-EGF) plays a critical role in wound healing by several mechanisms. It leads to the migration of fibroblasts, epithelial cells, and vascular endothelium in the proliferative phase; further, it stimulates the synthesis of DNA, RNA, protein, hyaluronic acid, and fibronectin $[8,9]$. EGF is known for its role in angiogenesis. Therefore, we hypothesized that the healing of vessels would also be influenced by the action of rh-EGF.

In our experiment, we hypothesized that chitosan would play a beneficial role in hemostasis and rh-EGF would enhance the effect of chitosan, which may account for the interaction of platelets with damaged tissues and the promotion of the healing effect of injured vessels.

\section{METHODS}

Forty-eight Sprague-Dawley rats, aged 10 to 12 weeks and weighing between 250 and $300 \mathrm{~g}$, were kept at room temperature $\left(23 \pm 1^{\circ} \mathrm{C}\right)$ and humidity of $55 \% \pm 5 \%$ with a $12: 12$ dark: light cycle under ambiguous light (150-300 Lux). All procedures were conducted in accordance with the guide for the care and use of laboratory animals of our center's Institutional Review Board. The rats were divided into the following four groups, and the experiment was performed on bilateral inguinal regions: group A: control (treated only with sterile gauze) $(\mathrm{n}=$ 24), group B: treated with gelatin sponge (Cutanplast, Mascia Brunelli, Milano, Italy) $(\mathrm{n}=24)$, group C: treated with "chitosan only" ( $\mathrm{n}=24)$, and group D: treated with "chitosan film impregnated with rh-EGF" ( $\mathrm{n}=24)$.
The chitosan-only film and the rh-EGF-impregnated chitosan film were prepared as follows: the EGF film was prepared to release $20 \mu \mathrm{g} /$ day/wound in the following manner: 4.5 weight/ volume $\%$ (w/v\%) chitosan, $4.5 \mathrm{w} / \mathrm{v} \%$ hydroxypropyl methylcellulose $50 \mathrm{cps}, 0.9 \mathrm{w} / \mathrm{v} \%$ hydroxypropyl methylcellulose 4,000 cps, $0.09 \mathrm{w} / \mathrm{v} \%$ pullulan, and $9 \mathrm{w} / \mathrm{v} \%$ glycerin were added to the distilled water and mixed with a stirrer. $1 \mathrm{mg} / \mathrm{mL}$ of EGF solution was added to the polymer solution and mixed in a cold chamber for 3 hours to form a homogeneous substance. The EGF-containing polymer solution was stored in a refrigerator for 24 hours in order to remove bubbles. It was poured onto the polyethylene terephthalate film liner and cast with a lab coater (LTE-S, Mathis, Switzerland). The casting thickness was 1.0 $\mathrm{mm}$. Next, the cast film was dried in hot air at $50^{\circ} \mathrm{C}$ for $30 \mathrm{~min}-$ utes. Then, the film was cut into $3 \mathrm{~cm} \times 3 \mathrm{~cm}$ samples, and an in vitro release test with a Franz-diffusion cell system was performed.

\section{Hemostasis model}

All prepared Sprague-Dawley rats were shaved and sterilized using betadine and alcohol. $10 \mathrm{mg} / \mathrm{kg}$ of xylazine-HCL (Rompun, Bayer Korea, Seoul, Korea) and $40 \mathrm{mg} / \mathrm{kg}$ of zoletil (Zoletil, Virbac, Carros, France) were injected intra-abdominally into these rats for anesthesia. A 3-cm-long longitudinal incision was made on both sides of the inguinal area to expose the femoral artery. Single 10-0 nylon sutures were used to mark the site of puncture for future histological study. Then, the femoral artery was punctured with an insulin syringe, and the bleeding was noted and then covered with a $1.5 \mathrm{~cm} \times 1.5 \mathrm{~cm}$ patch of the dressing material corresponding to the group to which the rat belonged. A 200 -g pendulum was then placed on top of the dressing to apply pressure and removed briefly after a 30 seconds interval to check whether the bleeding had stopped (Fig. 1). The time to reach immediate hemostasis was classified into four intervals: $30 \mathrm{sec}-$ onds, 60 seconds, 90 seconds, and more than 90 seconds (regarded as the failure of immediate hemostasis). After $90 \mathrm{sec}-$ onds, each dressing material patch remained over the puncture site and the incision site was closed primarily irrespective of whether immediate hemostasis was reached. After 1 day, the wound site was checked for hematoma collection.

\section{Histological changes in blood vessels}

After a week, the primary closed site was explored in the surviving rats. After removing the dressing materials from over the femoral artery, a specimen was taken from around the 10-nylon suture point. Each femoral artery specimen was fixed with formalin. Then, the histological change was observed by hematoxylin and eosin (H\&E) staining. 


\section{Fig. 1. Wound model using a Sprague-Dawley rat}

A puncture was made to the femoral artery and covered with a 1.5 $\mathrm{cm} \times 1.5 \mathrm{~cm}$ piece of dressing material according to each group. A 200-g pendulum was then placed on top of the dressing material to apply pressure and removed after a 30 -second interval to check whether the bleeding had stopped.

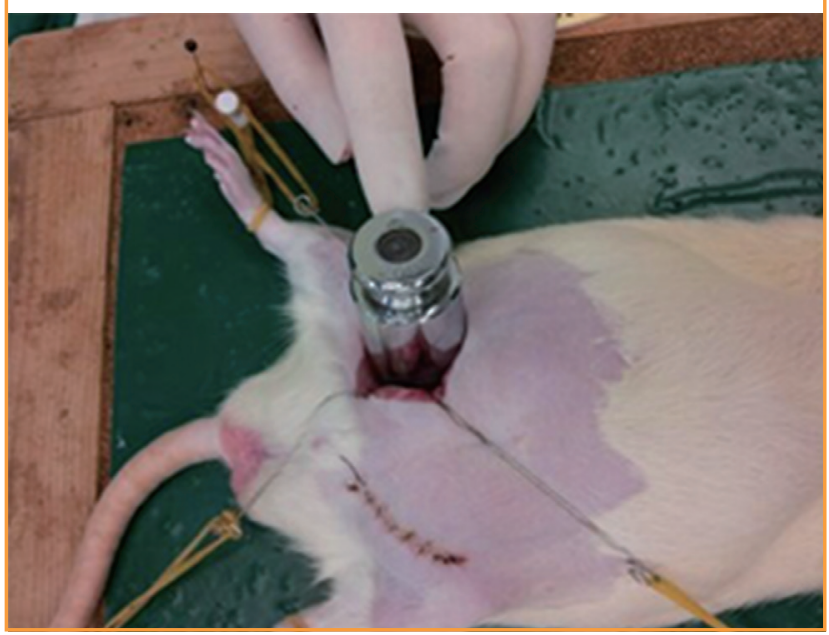

Table 1. Time needed for hemostasis

\begin{tabular}{lcccc|}
\hline & $\begin{array}{c}\mathbf{0 - 3 0} \\
\text { sec }\end{array}$ & $\begin{array}{c}\mathbf{3 1 - 6 0} \\
\text { sec }\end{array}$ & $\begin{array}{c}\mathbf{6 1 - 9 0} \\
\text { sec }\end{array}$ & $\begin{array}{c}>90 \\
\text { sec }\end{array}$ \\
\hline Group A, Guaze & 0 & 8 & 6 & 10 \\
Group B, Cutanplast & 7 & 10 & 6 & 1 \\
Group C, Chitosan & 6 & 9 & 6 & 3 \\
Group D, Chitosan \& rh-EGF & 7 & 9 & 6 & 2 \\
\hline The time needed to achieve immediate hemostasis is shown among the groups. \\
The experiment was considered a failure if immediate hemostasis was not \\
achieved within 90 seconds. The overall success of immediate hemostasis was \\
significant between group A and the others but not among groups B, C, and D. \\
rh-EGF, recombinant human epidermal growth factor. \\
\hline
\end{tabular}

For the statistical analysis, a chi-squared test was used to evaluate the statistical significance of the difference between the overall success rate and the success rates at 30 seconds and $60 \mathrm{sec}-$ onds (significant when $\mathrm{P}<0.05$ ). To compare group $\mathrm{D}$ with the others (groups A, B, and C), Bonferroni correction was utilized (significant when $\mathrm{P}<0.0167[=0.05 / 3]$ ).

\section{RESULTS}

In the hemostasis model, group $A$ failed to achieve immediate hemostasis in 10 puncture wounds, whereas groups B, C, and D had failures in 1 wound, 3 wounds, and 1 wound, respectively. In group A, none of the wounds achieved hemostasis before 30 $\mathrm{s}$ in contrast to the other groups, which achieved hemostasis in 7 (group B), 6 (group C), and 7 (group D) puncture wounds. Group A began to show hemostasis in the interval of 31-60 seconds, whereas other treatment groups showed more than half

\section{Fig. 2. Time needed for hemostasis}

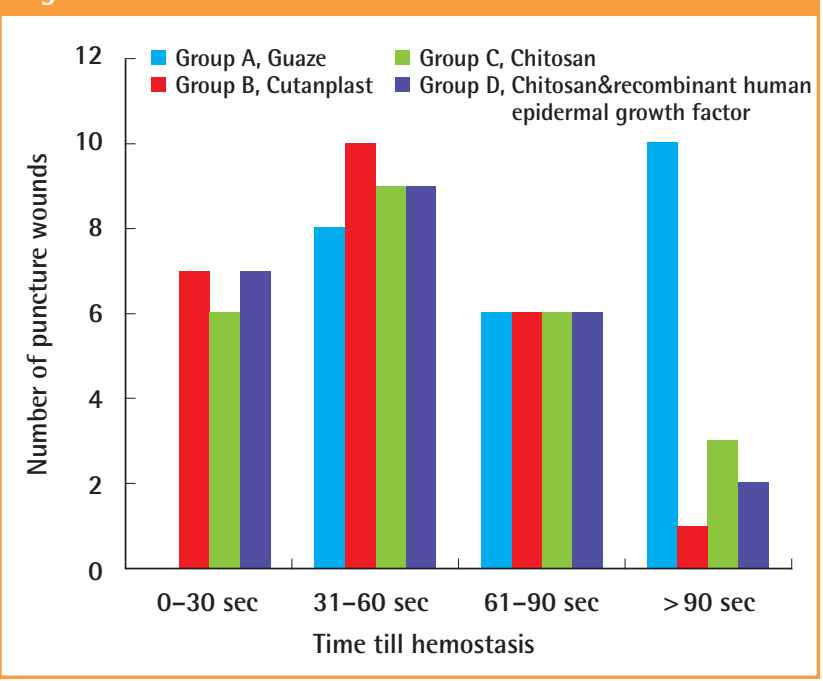

the specimens with hemostasis achieved by this time. Overall, the number of puncture wounds achieving immediate hemostasis within 90 seconds was 14, 23, 21, and 22 wounds, respectively, for groups A, B, C, and D; $56.5 \%$ for group A, $95.7 \%$ for group B, 87\% for group C, and $91.3 \%$ for group D (Table 1, Fig. $2)$. Groups B, C, and D showed a significant rate of immediate hemostasis as compared to group $A(P<0.05)$, but there were no significant differences among groups $\mathrm{B}, \mathrm{C}$, and $\mathrm{D}$. Two rats expired on day 1 in group $A$ with a massive hematoma in the inguinal region. There was no latent bleeding suggestive of hematoma in the other groups.

The histological evaluation showed distinctive changes among the groups. All specimens were gathered around the point of the 10-nylon suture near the puncture site. In group A, the continuity of the tunica adventitia in the puncture site was rarely observed in all the specimens. Further, the tunica media and the tunica intima had the tendency to show no continuity at all. In group B, the continuity of the vessel walls was seen, but the layer of tunica media was flimsy and some separation of the tunica adventitia layer was found. In group $\mathrm{C}$, the continuity of the tunica intima and the tunica media was clearly seen but showed separation from the tunica adventitia. Elastic lamellae were observed in the tunica media, but none of the smooth muscle cell nuclei were observed. In group $\mathrm{D}$, the overall vessel wall showed relatively good continuity. The tunica media's elastic lamellae and the smooth muscle cell nuclei clearly showed the healed vessel wall (Fig. 3).

\section{DISCUSSION}

Frequent debridement of large skin lesions may lead to blood loss and may prolong surgery because of the time spent on 


\section{Fig. 3. Microscopic observation of arteries (H\&E, ×100)}

\begin{abstract}
(A) Group A: the tendency toward discontinuity of the vessel wall at the puncture site can be seen (black arrow). (B) Group $B$ : the continuity of the vessel walls can be seen, but with thin layers of tunica media and some separation of the tunica adventitia layer (black arrow). (C) Group C: the layer of tunica media was flimsy, and some separation from the tunica adventitia was noted. Elastic lamella were observed in the tunica media, but smooth muscle cell nuclei were not observed (black arrow). (D) Group D: the overall vessel wall showed continuity. The tunica media's elastic lamellae and the smooth muscle cell nuclei were clearly found to show the most-healed vessel wall structure (black arrow).
\end{abstract}
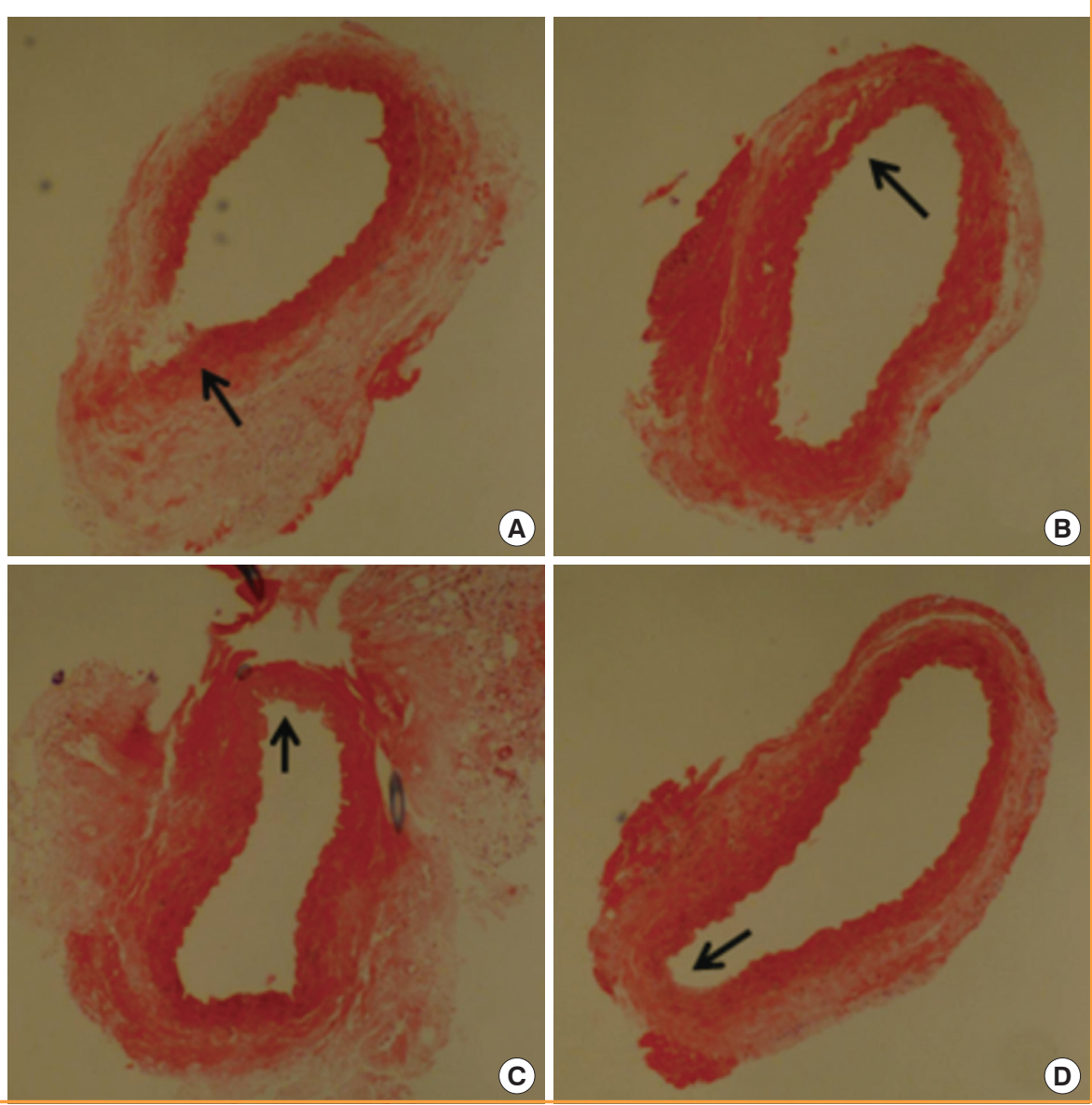

achieving hemostasis [10]. The same may be true for small debridement procedures that are done even at the bedside, and this may lead to prolonged bleeding, particularly in patients with chronic illness. Although portable electrocautery devices are available, often, the severity of small wounds may not require the routine use of such a device; further, such a device may not be readily available or economically feasible. Groenewold et al. [11] have shown that the use of epinephrine and fibrin sealant may reduce blood loss to some extent when a large amount of bleeding is anticipated while not impairing wound healing. However, the best method for hemostasis is yet to be determined.

Recently, a dressing material with a chitosan pad has shown to be effective after percutaneous procedures of the femoral artery [12]. It has been shown to decrease time-to-hemostasis as compared to a regular pad. Chitosan is a biodegradable, biocompatible, nontoxic, positively charged complex carbohydrate derived from the naturally occurring substance chitin [6]. Chitosan salt has been shown to have antimicrobial properties and to enhance wound healing [13-15]. According to multiple reviews, chitosan is a promising substance for achieving hemostasis after debridement. As seen in our study, the use of a chitosan film alone achieved immediate hemostasis similar to that achieved by a gelatin sponge, which has already been shown to be an effective agent for hemostasis [16].

The actual hemostasis occurred early after the puncture. The treatment groups began to show hemostasis within 30 seconds, while no hemostasis was noted in the gauze-treated group A during the same time interval. In most of the specimens, hemostasis was reached in the 31- to 60-second interval, whereas the control began to show about $30 \%$ hemostasis by this time. This observation suggests that early action is crucial to obtain meaningful hemostasis. In clinical situations, we do see situations where the patient comes back due to re-bleeding of the wound after debridement in an office setting. As seen in this study, failure to achieve good hemostasis in the early minutes of the puncture may lead to latent bleeding as shown in group A with massive hematoma collection leading to death in 2 rats. In contrast, groups B, C, and D did not show any latent bleeding. Thus, early hemostasis can lead to better control and reduced risk for latent bleeding.

Rh-EGF interacts with the EGF receptor on epidermal cells and fibroblasts. It is produced by platelets, macrophages, and 
monocytes, and its primary role is not only to stimulate epithelial cells to grow across the wound but also to act on fibroblasts and smooth muscle cells. Rh-EGF has been reported to significantly accelerate the epidermal regeneration of partial and fullthickness skin wounds in pigs, and continuous or prolonged exposure of rh-EGF can increase tensile strength in rat skin wounds [17]. EGF stimulates epithelialization in early human wound repair and is reported to enhance healing in chronic wounds such as diabetic foot ulcers [18]. We hypothesized that by combining rh-EGF with chitosan, we could augment the effect of vessel healing, which is the first step of wound healing. However, our past study showed that a combination of rh-EGF and chitosan did not result in a synergistic effect on the overall healing [15]. However, in this previous study, we did observe a significant increase in the rate of granulation when the study subjects were treated with the rh-EGF-combined chitosan film. This may suggest the potential effect against vascularization leading to increased granulation.

In this study, we found new data that revealed that the addition of rh-EGF to a chitosan film could enhance the healing of the artery walls. In the histologic analysis, group D showed sound and firm continuity on all layers of the vessel wall as compared to the other groups, particularly group $\mathrm{C}$ where endothelial healing was noted without any smooth muscle cell nuclei. One may presume that there will be injuries to the artery after the debridement of wounds. A sign of good debridement is the pinpoint bleeding from the wound bed. However, the effect of the early healing of arteries on the overall healing process is unknown. In our preliminary experiment with a healthy young porcine model, we observed that the overall healing of the rhEGF-impregnated chitosan film was not significant as compared to that of the chitosan-only film, at least in the case of acute healthy wounds. What we can confirm at this time is that rhEGF and chitosan do not impede each other's action and can enhance the healing of arteries while achieving acute hemostasis. The clinical use of rh-EGF in some countries has been shown to be beneficial for diabetic foot ulcers. The low concentration and the short acting half-life of rh-EGF have made it safe for topical use. However, we still need to understand the potential adverse effects that rh-EGF might have depending on its dosage. Although some papers have been published on the positive role of rh-EGF against cancer formation, there are still debates related to this issue [19].

In conclusion, due to its abovementioned characteristics, chitosan, which had a positive charge and induced platelet adhesion and aggregation, showed effective hemostatic potential. Further, the mix of rh-EGF and chitosan did not interfere with chitosan's hemostatic capabilities. We also observed better heal- ing in vessel walls, which was promoted by hemostasis, when rhEGF was added to chitosan. On the basis of these positive findings, we believe that further research is needed to evaluate the potential use of rh-EGF-impregnated chitosan over difficult wounds like chronic diabetic ulcerations.

\section{REFERENCES}

1. Schafer AI. Approach to the patient with bleeding and thrombosis. In: Goldman L, Ausiello DA, Arend W, editors. Cecil medicine. 23rd ed. Philadelphia: Saunders Elsevier; 2008. p.1286-8.

2. Sabino L, Andreoni C, Faria EF, et al. Evaluation of renal defect healing, hemostasis, and urinary fistula after laparoscopic partial nephrectomy with oxidized cellulose. J Endourol 2007;21:551-6.

3. Li Q, Dunn ET, Grandmaison EW, et al. Applications and properties of chitosan. J Bioact Compat Polym 1992;7:37097.

4. Gebelein CG, Dunn RL; American Chemical Society Meeting. Progress in biomedical polymers. New York: Plenum Press; 1990.

5. Chou TC, Fu E, Wu CJ, et al. Chitosan enhances platelet adhesion and aggregation. Biochem Biophys Res Commun 2003;302:480-3.

6. Klokkevold PR, Lew DS, Ellis DG, et al. Effect of chitosan on lingual hemostasis in rabbits. J Oral Maxillofac Surg 1991; 49:858-63.

7. Klokkevold PR, Fukayama H, Sung EC, et al. The effect of chitosan (poly-N-acetyl glucosamine) on lingual hemostasis in heparinized rabbits. J Oral Maxillofac Surg 1999;57:49-52.

8. Santa Maria PL, Redmond SL, Atlas MD, et al. The role of epidermal growth factor in the healing tympanic membrane following perforation in rats. J Mol Histol 2010;41:309-14.

9. Hom DB, Maisel RH. Angiogenic growth factors: their effects and potential in soft tissue wound healing. Ann Otol Rhinol Laryngol 1992;101:349-54.

10. Niemi TT, Taxell C, Rosenberg PH. Comparison of the effect of intravenous ketoprofen, ketorolac and diclofenac on platelet function in volunteers. Acta Anaesthesiol Scand 1997;41:1353-8.

11. Groenewold MD, Gribnau AJ, Ubbink DT. Topical haemostatic agents for skin wounds: a systematic review. BMC Surg 2011;11:15.

12. Arbel J, Rozenbaum E, Reges O, et al. USage of chitosan for Femoral (USF) haemostasis after percutaneous procedures: a comparative open label study. EuroIntervention 2011;6: 1104-9. 
13. Burkatovskaya M, Castano AP, Demidova-Rice TN, et al. Effect of chitosan acetate bandage on wound healing in infected and noninfected wounds in mice. Wound Repair Regen 2008;16:425-31.

14. Burkatovskaya M, Tegos GP, Swietlik E, et al. Use of chitosan bandage to prevent fatal infections developing from highly contaminated wounds in mice. Biomaterials 2006;27: 4157-64.

15. Hong JP, Kim YW, Lee SK, et al. The effect of continuous release of recombinant human epidermal growth factor (rhEGF) in chitosan film on full thickness excisional porcine wounds. Ann Plast Surg 2008;61:457-62.

16. Cho KS, Shin SK, Lee JH, et al. The efficacy of Cutanplast nasal packing after endoscopic sinus surgery: a prospective, randomized, controlled trial. Laryngoscope 2013;123:564-8.

17. Brown GL, Curtsinger L 3rd, Brightwell JR, et al. Enhancement of epidermal regeneration by biosynthetic epidermal growth factor. J Exp Med 1986;163:1319-24.

18. Hong JP, Jung HD, Kim YW. Recombinant human epidermal growth factor (EGF) to enhance healing for diabetic foot ulcers. Ann Plast Surg 2006;56:394-8.

19. Choi J, Moon SY, Hong JP, et al. Epidermal growth factor induces cell death in the absence of overexpressed epidermal growth factor receptor and ErbB2 in various human cancer cell lines. Cancer Invest 2010;28:505-14. 